\title{
Consistency of individual differences in brightness perception
}

\author{
RICHARD W. BOWEN \\ Loyola University of Chicago, Chicago, Illinois
}

\begin{abstract}
In previous studies, we have measured individual differences in simultaneous brightness discrimination between a long-duration ( $500 \mathrm{msec}$ or more) comparison pulse and a set of briefer test pulses of the same luminance. Some observers judge the comparison pulse to be brighter than all test pulses in a range from 20 to $320 \mathrm{msec}$. Other observers exhibit temporal brightness enhancement: a test pulse of $80 \mathrm{msec}$ for our stimulus conditions is judged brighter than the comparison pulse. Individual differences depend upon the temporal asynchrony between test and comparison pulses. Observers designated Type A show temporal brightness enhancement for both simultaneous onset of test and comparison pulse and for simultaneous offset of the pulses. Type B observers exhibit brightness enhancement for simultaneous offset of pulses but not for simultaneous onset. Type $\mathrm{C}$ observers do not exhibit brightness enhancement effects. Here we use both short (80 msec) and long $(640 \mathrm{msec})$ pulses as the comparison stimuli. We replicate our previous pattern of individual differences for the long comparison stimulus. For the short comparison stimulus, brightness discrimination functions for the three classes of observers are exactly predicted from the patterns obtained with the conventional long comparison pulse. This confirms the generality of individual differences in pulse brightness perception and shows that they do not depend upon the particular stimulus conditions selected for the discrimination task.
\end{abstract}

When observers are confronted with a brightness discrimination between a long-duration ( $500 \mathrm{msec}$ or more) comparison pulse and a series of briefer test pulses of the same luminance, two outcomes have been observed (Bowen \& Markell, 1980). Some observers consistently judge the longer pulse as brighter, with a probability that decreases to an asymptote of 0.5 as test pulse duration increases. That is, a monotonic increase in brightness with pulse duration can be inferred from the discriminations. Other observers show a nonmonotonic brightness-duration relation: test pulses of intermediate duration (e.g., $80 \mathrm{msec}$ ) are judged brighter than the longer comparison pulse. This result indicates a temporal brightness enhancement (Broca-Sulzer) effect; we infer that brightness is maximum for pulses of intermediate duration and less for longer or shorter pulses.

If the asynchrony between test and comparison pulses is manipulated, with pulses either coming on together (simultaneous onset) or going off together (simultaneous offset), the population of observers may be further classified into three groups. Observers designated Type A show brightness enhancement (nonmonotonic discrimination) for both simultaneous onset and offset conditions. Type B observers show a brightness enhancement effect for the simultaneous offset condition and a monotonic discrimination function for the condition of simultaneous on-

The research was supported by National Science Foundation Grant BNS 81-11366.

The author's mailing address is: Department of Psychology, Loyola University of Chicago, Chicago, IL 60626. set. Type $\mathrm{C}$ observers show monotonic discrimination for both asynchrony conditions, and give no evidence of the brightness enhancement effect.

This paper describes an experiment that tested the generality of this pattern of individual differences. The issue addressed was whether these differences were limited to the conditions of discrimination under which they were first observed, that is, one of a series of short test pulses compared on each experimental trial with a long comparison pulse. Here we employed both a short comparison pulse $(80 \mathrm{msec})$ and a long one $(640 \mathrm{msec})$. (If brightness enhancement occurs, an 80-msec pulse will have the greatest brightness under our stimulus conditions; Bowen, 1984.) We asked whether the pattern of results obtained with a short comparison pulse could be exactly predicted from the well-established pattern determined with the long comparison pulse.

\section{METHOD}

\section{Observers}

The observers were 46 undergraduate students fulfilling a course requirement for general psychology. The observers were screened for stereopsis and for visual acuity of $6 / 6$ or better.

\section{Apparatus and Procedure}

The apparatus, a haploscopic optical projection system, has been completely described elsewhere (Bowen \& Markell, 1980; Bowen, Sekuler, Owsley, \& Markell, 1981). It generated two circular targets (34-min diam) separated by $2^{\circ} 50^{\prime}$; a binocular fixation target was positioned midway between the targets, which were presented separately to right and left eyes. The targets could be pulsed with 
any desired timing relation under computer control. The stimulus display was viewed in darkness.

The following procedure was employed. On each trial, a test pulse and a comparison pulse were presented at the target locations. Positions of test pulses were varied randomly. All pulses had a luminance of $100 \mathrm{~cd} / \mathrm{m}^{2}$. Test pulse durations of $20,40,80,160$, and $320 \mathrm{msec}$ were used. Test and comparison pulses were presented with an asynchrony of either simultaneous onset or simultaneous offset of pulses. The experiment was conducted in two blocks of 200 trials with a short break between blocks. In one block of trials, the comparison pulse was short $(80 \mathrm{msec})$, and in the other it was long $(640 \mathrm{msec})$. The observers were randomly designated to run either with the short or the long comparison in the first block of trials, with the remaining comparison stimulus run in the second block of trials. A block consisted of a completely randomized combination of five test pulse durations, two pulse asynchronies, and 20 trials (10 with test pulse on the left of the stimulus display, 10 with it on the right). The observer initiated a trial by depressing a button and reported which pulse appeared brighter, left or right, by pressing one of two other buttons.

As an aid to visualizing the temporal relations between test and comparison pulses for the conditions studied, Figure 1 gives a schematic depiction of these relations for the long comparison pulse (top half of figure) and the short comparison pulse (bottom half of figure). Simultaneous onset and offset conditions are represented for just two test pulse durations ( 80 and $320 \mathrm{msec}$ ).

\section{RESULTS AND DISCUSSION}

Figure 2 gives the results as plots of the mean percentage of trials in which the comparison pulse was judged brighter as a function of the duration of the test pulse. The left three panels give data for a comparison pulse of $640 \mathrm{msec}$, and the right panels show results for a com-

\section{COMPARISON PULSE - 640 MS}
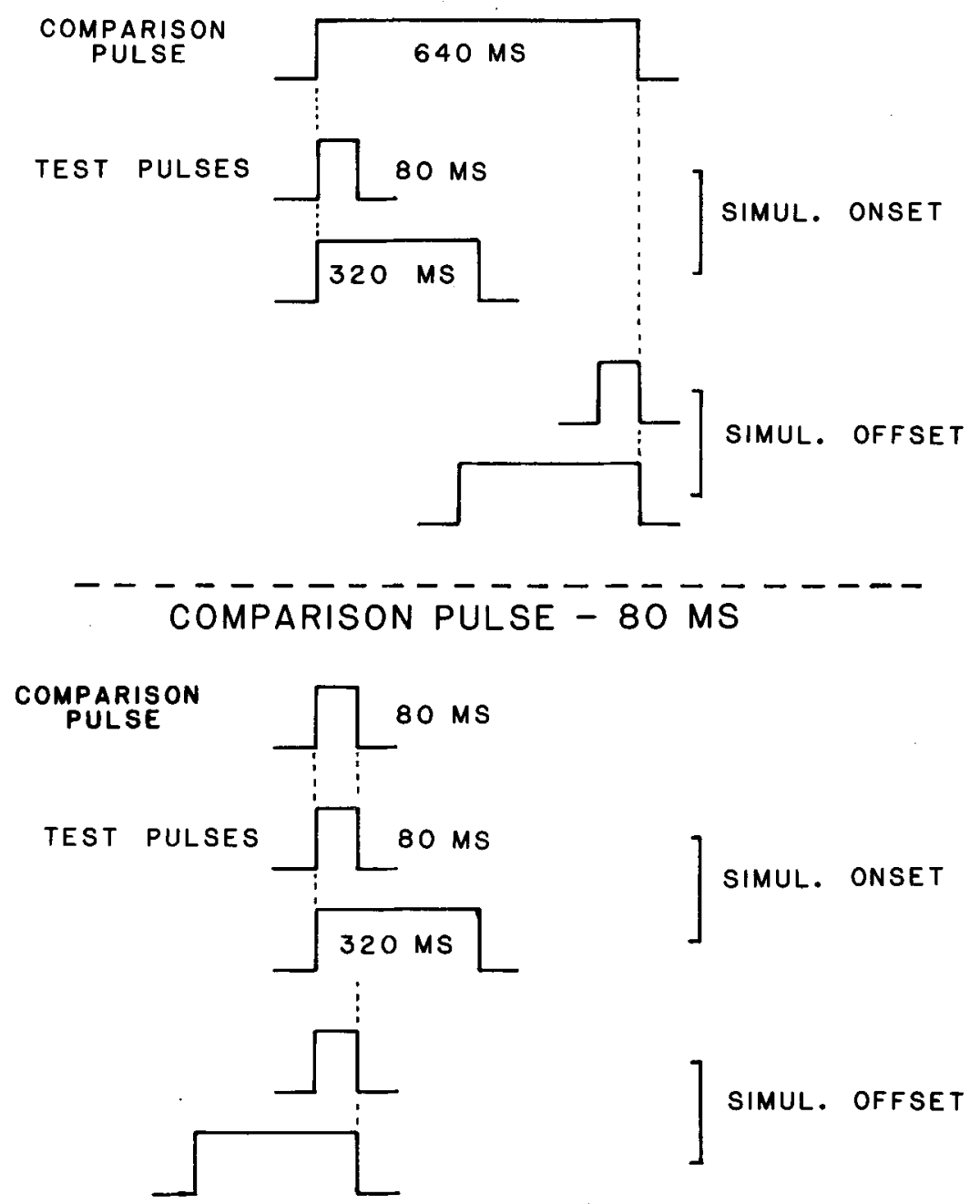

Figure 1. Schematic representation of the temporal relations between test pulse and comparison pulse for the simultaneous onset and simultaneous offset asynchrony conditions. Top of figure: 640-msec comparison pulse; bottom of figure: 80-msec comparison pulse. Temporal relations are illustrated for test pulses of 80 and $320 \mathrm{msec}$, although in the experiment test pulses of 20, 40, 80, 160, and 320 msec were employed. 


\section{COMPARISON - 640 MS COMPARISON - 80 MS}

TYPE A $\quad(N=15)$
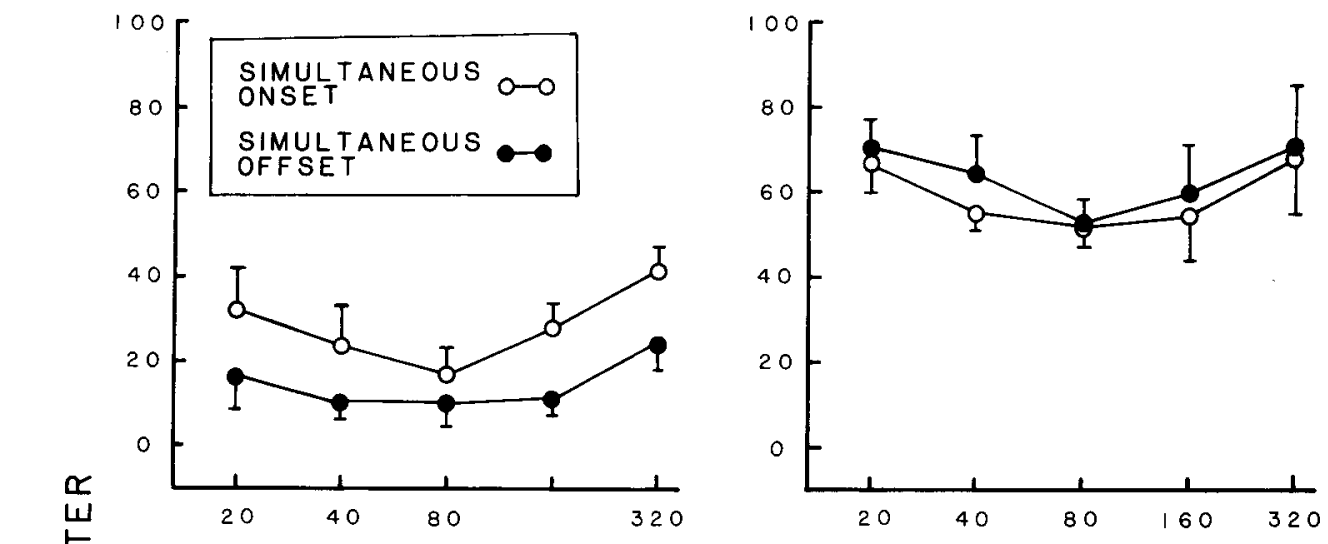

$\omega$

$\frac{1}{0}$

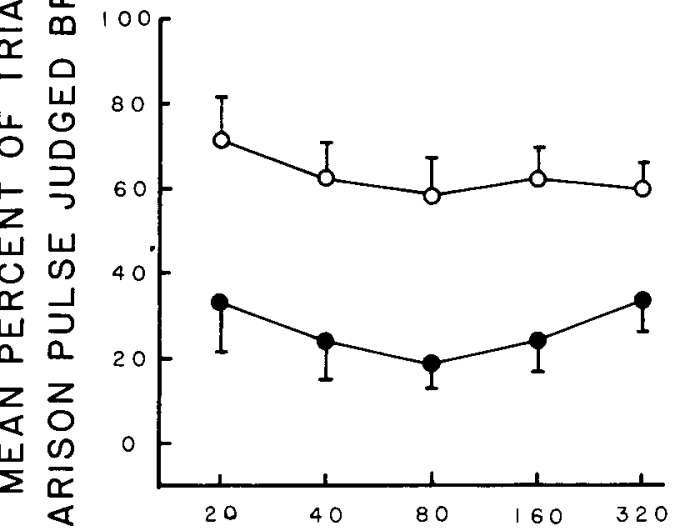

TYPE B $\quad(N=17)$

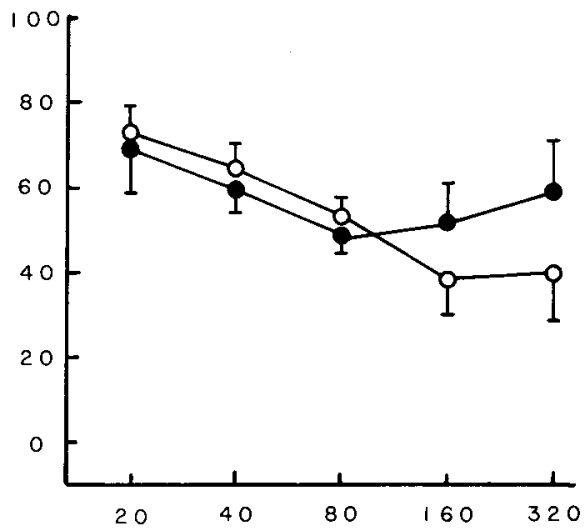

TYPE C $\quad(N=\mid 4)$
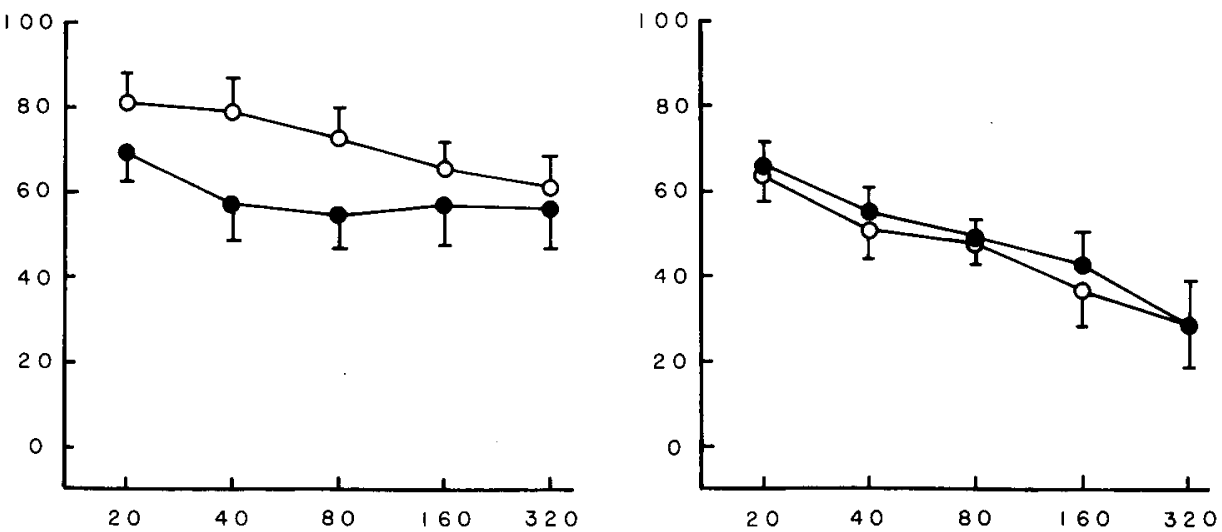

\section{DURATION OF TEST PULSE (MS)}

Figure 2. Plots of the mean percentage of trials on which the comparison pulse was judged brighter as a function of the duration of the test pulse. Left panels are for a comparison pulse of 640 msec; right panels are for a comparison pulse of 80 msec. Type A (top), Type B (middle), and Type C (bottom) observers were categorized on the basis of functions for the 640 -msec comparison pulse. See text. 
parison of $80 \mathrm{msec}$. Open symbols indicate the simultaneous-onset asynchrony condition; filled symbols indicate the simultaneous-offset condition. Error bars indicate $95 \%$ confidence intervals.

The observers were categorized as Type A, B, or C on the basis of the data for the 640-msec comparison pulse, as in previous studies (see Bowen, 1984). The criterion for the occurrence of brightness enhancement was a binomial one (Bowen \& Markell, 1980): $30 \%$ or less of " 640 -msec comparison pulse judged brighter" at any pulse duration was taken as evidence of brightness enhancement, since 6 trials out of 20 is significantly lower $(p<.057)$ than $50 \%$ in a binomial distribution, assuming a probability of .5 for responding "comparison pulse brighter." Among the 46 observers, 15 could be classified as Type A, 17 as type B, and 14 as Type C.

With the 640-msec comparison stimulus, functions for Type A observers (top panel, left side) at both asynchrony conditions have a minimum at $80 \mathrm{msec}$ (a brightness enhancement effect). This pattern of discrimination completely replicates previous work (e.g., Bowen, 1984), and it implies that the 80-msec test pulse appeared brighter than all shorter or longer test pulses, since it was judged brighter than the 640-msec comparison on the greatest percentage of trials. Now, if an 80 -msec pulse is itself used as a comparison pulse for Type A observers, an 80-msec test pulse should still be the minimum of the function, but the minimum level should be near $50 \%$ ( $80 \mathrm{msec}$ compared with $80 \mathrm{msec}$ ) rather than near $0 \%(80 \mathrm{msec}$ compared with $640 \mathrm{msec}$ ). From the data of Figure 2 (top panel, right side), this is exactly what was obtained for Type A observers.

As in previous studies, Type B observers give a minimum at $80 \mathrm{msec}$ for simultaneous offset conditions, indicating brightness enhancement relative to the $640-\mathrm{msec}$ comparison stimulus (middle panel, left side). The function for simultaneous onset with the 640-msec comparison is elevated above $50 \%$ and decreases toward $50 \%$ with increasing test-pulse duration. This implies that increasing test-pulse duration produces progressively increasing brightness. Therefore, under the simultaneous onset condition, the 80-msec comparison pulse should be judged brighter than shorter pulses (the function should be above $50 \%$ ) but should be judged dimmer than longer pulses (the function should be below $50 \%$ ). For simultaneous offset, the 80-msec comparison pulse should yield the same function as was obtained for Type A observers with this asynchrony, that is, a local minimum at the $50 \%$ level for an 80-msec test pulse. The data of Figure 2 (middle panel, right side) clearly follow the pattern predicted for Type B observers with a short comparison stimulus.

Finally, for Type C observers, the 640 -msec pulse is judged brighter than all test pulses with functions at both asynchronies declining toward $50 \%$ (bottom panel, left side). With an 80-msec comparison pulse, one would predict monotonic functions passing through $50 \%$ for an 80-msec test pulse, just as with the simultaneous onset data for Type B observers. The data (lower panel, right side) again follow the predicted pattern.

Figure 2 presents composite data; we also analyzed data for individual observers. Given that an observer was classified as Type $\mathrm{A}, \mathrm{B}$, or $\mathrm{C}$ with the long-duration comparison stimulus (see Method above), we determined the classification for the individuals on the basis of their data for the short-duration comparison. The criterion for the occurrence of brightness enhancement was whether the 80-msec comparison pulse was judged significantly brighter than test stimuli of 160 or $320 \mathrm{msec}$ duration (70\% or greater judgments of "comparison brighter," equivalent to the binomial criterion used with the longduration comparison). (For test pulses of 20 and $40 \mathrm{msec}$, we expect the 80-msec comparison to be judged brighter based simply upon temporal summation; Bowen \& Markell, 1980.) Classification of observers as A, B, or $C$ was, as usual, based upon occurrence of brightness enhancement for the two asynchrony conditions.

Table 1 summarizes the analysis for individual observers. There is a clear tendency for observers classified as a given type with the long-duration comparison to be so classified with the short-duration comparison. This was true for 10 of 15 Type A (66\%), 12 of 17 Type B (71\%), and 14 of 14 Type C observers. A Pearson chi-square test of association was conducted on the frequency data of Table 1 ; the chi-square value was equal to $44.11(d f=4)$, significant at the .01 level. We reject the hypothesis of independence between comparison conditions, and claim significant statistical association between the distributions of observer types for these conditions.

Our results demonstrate generality in the individual differences in brightness perception. The same underlying brightness-versus-test-pulse-duration relation (monotonic or nonmonotonic) can be inferred for each type of observer (A, B, and C) whether the comparison stimulus is long, and of asymptotic brightness, or short, and thus either at peak brightness (for A and B observers) or intermediate brightness (for $\mathrm{C}$ observers). The effect of pulse asynchrony (simultaneous onset vs. simultaneous onset) in establishing the form of this brightness-duration relation is likewise the same for both short and long comparison pulses. The present data do not, however, help explain why the pulse asynchrony conditions generate these individual differences. A puzzle, for example, is the

Table 1

Classification of Observers (A, B, C) for Short-Duration Comparison Stimulus Given Their Classification for the Long-Duration Comparison Stimulus

\begin{tabular}{crrr}
\hline $\begin{array}{c}\text { Type of Observer } \\
\text { as Classified with }\end{array}$ & \multicolumn{2}{c}{$\begin{array}{c}\text { Frequency of Type-of-Observer- } \\
\text { Short-Duration Comparison }\end{array}$} \\
\cline { 2 - 4 } Long-Duration Comparison & A & B & C \\
\hline A $(N=15)$ & 10 & 3 & 2 \\
B $(N=17)$ & 2 & 12 & 3 \\
C $(N=14)$ & 0 & 0 & 14 \\
\hline
\end{tabular}


behavior of Type B observers, for whom we infer a different brightness-duration relation for simultaneous onset of pulses versus simultaneous offset. The present data do not speak to why Type B observers apparently adopt different perceptual criteria in these two cases (Bowen, 1984), but they do demonstrate that the effect of pulse asynchrony on Type B observers is consistent independent of the duration of the comparison event.

Consistency across comparison conditions did not necessarily have to occur. The originally observed pattern of individual differences might have been due to the particular choice of stimulus conditions or discrimination paradigm. For example, Bernstein, Proctor, Proctor, and Schurman (1973) demonstrated that in a metacontrast masking situation, a given observer may show a shift in criterion for judging the brightness of a masked stimulus, depending on the stimulus asynchrony. With simultaneous presentation of mask and test stimuli, the criterion is comparative (the masking stimulus provides a frame of reference); the judgment becomes absolute as the delay between mask and test is increased. In our previous studies, the presence of a long-duration comparison stimulus on every trial could have generated such a frame of reference. Different classes of observers might have adopted different varying criteria for brightness of test pulses relative to the brightness of the constant frame of reference. However, the behavior of, for example, Type $\mathrm{C}$ observers is apparently not determined by the presence of a long comparison pulse on every experimental trial. We infer that brightness increases continuously with increasing pulse duration for Type $\mathrm{C}$ observers, and this inference is valid whether brief pulses are compared with intermediate pulses ( $20 \mathrm{msec}$ vs. $80 \mathrm{msec}$ ) or intermediate pulses are compared with long pulses $(80 \mathrm{msec}$ vs. $640 \mathrm{msec}$ ). In general, the fact that a predictable pattern of individual differences occurs with a short comparison gives us confidence that the differences are actually based on differences in brightness among test pulses rather than on any peculiarities of the discrimination task or the stimulus with which all test pulses are compared.

\section{REFERENCES}

Bernstein, I. H., Proctor, J. D., Proctor, R. W., \& Schurman, D. L. (1973). Metacontrast and brightness discrimination. Perception \& Psychophysics, 14, 293-297.

BoweN, R. W. (1984). Temporal brightness enhancement: Studies of individual differences. Perception \& Psychophysics, 36, 401-408.

Bowen, R. W., \& MARKelL, K. A. (1980). Temporal brightness enhancement studied with a large sample of observers: Evidence for individual differences in brightness perception. Perception \& Psychophysics, 27, 465-476.

Bowen, R. W., Sekuler, R., Owsley, C. J., \& Markell, K. A. (1981). Individual differences in brightness perception. Perception \& Psychophysics, 30, 587-593.

(Manuscript received December 30, 1985; revision accepted for publication June 13, 1986.) 